\title{
System Control Design of Autopilot and Speed Pilot for a Patrol Vessel by Using LMIs
}

\author{
A. Alessandri \& S. Donnarumma \& S. Vignolo \\ Department of Mechanical, Power, Industrial and Transport Engineering (DIME) \\ University of Genoa, Genoa, Italy
}

M. Figari \& M. Martelli

Department of Marine, Electronic, Electrical and Telecommunications Engineering (DITEN)

University of Genoa, Genoa, Italy

R. Chiti \& L. Sebastiani

SEASTEMA SpA

Genova, Italy.

ABSTRACT: Heading and speed control for a patrol vessel is addressed by using simple PID regulators. The selection of the PID parameters for both controllers is accomplished by using decoupled linearized model of the original motion equations and LMIs as a design tool. The effectiveness of the resulting controllers is validated on the original dynamic equations and with the presence of external disturbances such as wind, waves, and current.

\section{INTRODUCTION}

Automatic steering of ships has been a goal of marine engineering for many years. Milestones such as reduced manning, increasing fuel costs and performance optimization has led to innovative design approaches (Roberts, Sutton, Zirilli, \& Tiano 2003) and (Fuller 1979). Modern vessels require higher and higher levels of automation on board to guarantee enhanced performances in various environmental conditions. In this context, more sophisticated autopilots and speed pilots enable the ship masters to operate the vessel with the desired heading and speed. Toward this end, an approach the design of both autopilot and speed pilot is presented. Specifically, we refer as case study to a patrol vessel of the Italian Coast Guard fitted with two screw propellers and two rudders.

In order to guarantee both path keeping and small induced drag, the controller has to avoid high rudder angle requirements as well as to take into account rudder rotation rate bounds (Tzeng \& Lin 2000). Indeed, large oscillations of the rudder must be prevented for two main reasons, i.e., reduction of mechanical stress and generation of useless high-frequency motion of the rudder (Källström 2000). Robustness to external disturbances is a fundamental issue to account for (Lauvdal \& Fossen 1998).
As compared with the previous literature, a simpler approach is presented for the design of regulators for heading and speed since a reduced number of parameters such as PID (proportional, integral, and derivative) controllers is imposed as a strict requirement to facilitate the tuning during sea tests. However, a novelty with respect to the state-of-art is proposed as the controllers synthesis is obtained by using Linear Matrix Inequalities (LMIs). LMI techniques allow one to solve a large number of control problems, estimation, and stability analysis that may be formulated as convex problems (Boyd, El Ghaoui, Feron, \& Balakrishnan 1994). In particular, an LMI solution of the gain assignment for feedback linear time-invariant systems is considered here that is well-suited to designing PID controllers for the autopilot and speed pilot.

The synthesis of regulators is based on simplified dynamic equations obtained by decoupling surge and yaw motions. More specifically, the equations are linearized around the desired advance speed after neglecting environmental disturbances. The resulting controllers are then validated by means of a complete simulation model where all involved motions are coupled through the original dynamical equations with environmental disturbances explicitly taken into account.

The layout of the paper is as follows. In section 2 
the dynamical model of the vessel is outlined. In section 3 the LMI approach is applied to single out the PID controllers for heading and advance speed. Sections 4 and 5 are devoted to the description of environmental and actuated forces. Section 6 presents simulation results. Finally, in section 7 conclusions and future developments are discussed.

\section{VESSEL MODELLING}

Following well known notations (Fossen 2002), let us denote by $\boldsymbol{\eta}:=[x, y, \psi]^{\top} \in \mathbb{R}^{3}$ the array containing the position and the orientation of the vessel w.r.t. the earth-fixed frame, and by $\boldsymbol{\nu}:=[u, v, r]^{\top} \in \mathbb{R}^{3}$ the array containing the linear and angular velocity components w.r.t. the body-fixed basis. Then the motion equations of the vessel can be expressed as follows:

$\dot{\boldsymbol{\eta}}=\mathbf{R}(\psi) \boldsymbol{\nu}, \quad \mathbf{R}(\psi)=\left[\begin{array}{ccc}\cos \psi & -\sin \psi & 0 \\ \sin \psi & \cos \psi & 0 \\ 0 & 0 & 1\end{array}\right]$,

$\mathbf{M} \dot{\boldsymbol{\nu}}+\mathbf{C}(\boldsymbol{\nu}) \boldsymbol{\nu}-\mathbf{D}_{0} \boldsymbol{\nu}_{r}-\mathbf{D}\left(\boldsymbol{\nu}_{r}\right) \boldsymbol{\nu}_{r}=\boldsymbol{\tau}_{D}+\boldsymbol{\tau}_{E}$,

where $\boldsymbol{\tau}:=[X, Y, N]^{\top} \in \mathbb{R}^{3}$ is the array expressing the resultant longitudinal and lateral forces and the resultant moment (evaluated w.r.t. midship) acting on the vessel. The subscript $D$ refers to delivered forces while $E$ regards environmental disturbances; $\boldsymbol{\nu}_{r}:=\boldsymbol{\nu}-\boldsymbol{\nu}_{c}$ denotes the relative velocity w.r.t. to the current. $\mathbf{M}, \mathbf{C}, \mathbf{D}_{0}$, and $\mathbf{D}$, in equation (2), are the inertia and added masses, Coriolis-centripetal and, linear/nonlinear damping matrices, respectively. They are defined as follows:

$$
\begin{aligned}
& \mathbf{M}=\left[\begin{array}{ccc}
m-X_{\dot{u}} & 0 & 0 \\
0 & m-Y_{\dot{v}} & m x_{G} \\
0 & m x_{G} & I_{z}-N_{\dot{r}}
\end{array}\right], \\
& \mathbf{C}=\left[\begin{array}{ccc}
0 & 0 & -m\left(x_{G} r+u\right) \\
0 & 0 & m u \\
m\left(x_{G} r+u\right) & -m u & 0
\end{array}\right], \\
& \mathbf{D}_{0}=\left[\begin{array}{ccc}
0 & 0 & 0 \\
0 & Y_{v} & Y_{r} \\
0 & N_{v} & N_{r}
\end{array}\right], \\
& \mathbf{D}(\boldsymbol{\nu})=\left[\begin{array}{ccc}
R(u) & X_{v v} v & X_{v r} v+ \\
0 & Y_{v|v|}|v| & Y_{r|v|}|v|+ \\
& & +Y_{r|r|} r|r|+ \\
& & +Y_{v r r} v r \\
0 & N_{v|v|}|v|+ & N_{r|r|}|r|+ \\
& +N_{r|v|}|v| & +N_{v r r} v r
\end{array}\right],
\end{aligned}
$$

where $X_{\dot{u}}<0, Y_{\dot{v}}<0$, and $N_{\dot{r}}<0$ are the only available zero frequency added masses coefficients; $R(u)$

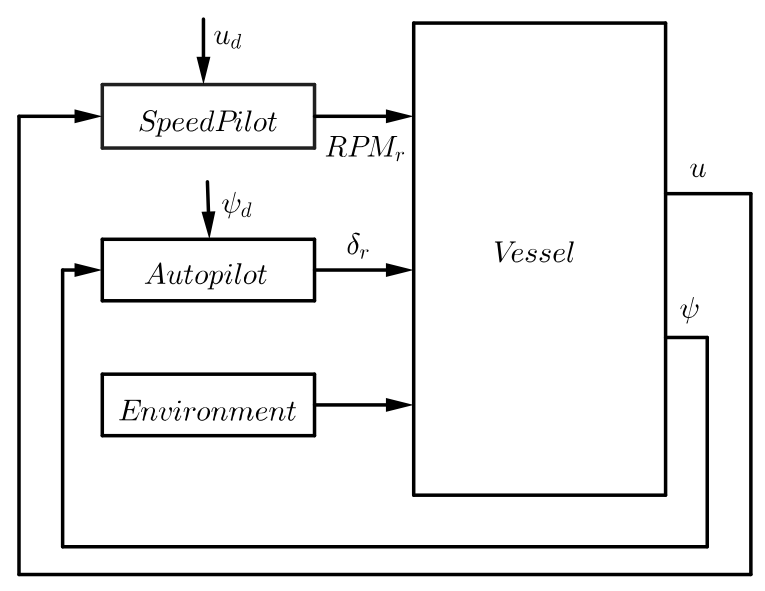

Figure 1: Speed pilot and autopilot main plants.

is the drag coefficient; coefficients in matrices (5) and (6) are defined in accordance with (Ankudinov, Kaplan, \& Jacobsen 1993). Practical applications, concerning low speed and high speed vessels modeling, can be found in (Altosole, Boote, Brizzolara, \& Viviani 2013) and (Martelli, Viviani, Altosole, Figari, \& Vignolo 2014), respectively.

\section{DESIGN OF THE CONTROL SYSTEM}

In figure 1 the main plant logic is outlined. The control system is composed by two distinguished controllers: an autopilot for heading control and a speed pilot to keep the surge velocity constant. In both cases, an approach to the synthesis of PID controllers by using LMIs is presented. We will firstly illustrate the design of the speed pilot and then that of the autopilot.

\subsection{Speed Pilot}

The surge equation is decoupled from sway and yaw motions and it is linearized around the desired advance velocity $u_{d}$. Since, in this case of study, $u_{d}$ is close to the design speed and the navigationguidance law keeps the pitch angle constant over a large speed range, the only available degree of freedom for propulsion management is given by the shaft speed $n_{p}$. Under the stated assumption, the linearized surge equation assumes the following expression:

$$
\left(m-X_{\dot{u}}\right) \dot{u}+R\left(u_{d}\right) u=b n_{p},
$$

where $b$ is a suitable coefficient linking the thrust of both the propellers to the shaft speed. In order to model the dynamical behavior of the propulsion line, a first-order differential equation is added to the system (7):

$\dot{u}=-\frac{R\left(u_{d}\right)}{m-X_{\dot{u}}} u+\frac{b}{m-X_{\dot{u}}} n_{p}$,

$\dot{n}_{p}=\tau_{p}\left(n_{r}-n_{p}\right)$, 
where $n_{r}$ is the desired shaft speed and $\tau_{p}$ is a positive time constant accounting for the response of the propulsion line.

The PID regulator can be represented as a transfer function $G(s)$ given by

$G(s)=K_{P}+K_{I} \frac{1}{s}+K_{D} s$,

with the scalar parameters $K_{P}, K_{I}$, and $K_{D}$ to be suitably chosen in such a way to ensure closed-loop stability. In practice, the control signal is as follows:

$n_{r}(t)=K_{P} e(t)+K_{I} \int_{0}^{t} e(\xi) \mathrm{d} \xi+K_{D} \dot{e}(t)$,

where $e:=u_{d}-u$ is the speed error. Thus, the resulting closed-loop dynamics is

$$
\begin{aligned}
\dot{x}_{1} & =a_{11} x_{1}+a_{12} x_{2}, \\
\dot{x}_{2} & =a_{22} x_{2}+b\left(K_{P}\left(u_{d}-x_{1}\right)+K_{I} x_{3}+\right. \\
& \left.-K_{D}\left(a_{11} x_{1}+a_{12} x_{2}\right)\right) \\
\dot{x}_{3} & =u_{d}-x_{1}
\end{aligned}
$$

where

$$
\begin{aligned}
& a_{11}:=-\frac{R\left(u_{d}\right)}{m-X_{\dot{u}}}, \quad a_{12}:=\frac{b}{m-X_{\dot{u}}}, \\
& a_{22}:=\tau_{p} .
\end{aligned}
$$

After defining

$$
\begin{aligned}
K_{1} & :=b K_{P}-b K_{D} a_{11}, \quad K_{2}:=b K_{D} a_{12}, \\
K_{3} & :=-b K_{I},
\end{aligned}
$$

the previous differential equation can be equivalently written as follows:

$\dot{\boldsymbol{x}}=(\mathbf{A}-\mathbf{B K}) \boldsymbol{x}+\mathbf{E} u_{d}$,

where $\boldsymbol{x}=\left[u, n_{p}, e\right]^{\top} \in \mathbb{R}^{3}$ collects all the state variables,

$$
\begin{aligned}
\mathbf{A} & :=\left[\begin{array}{ccc}
a_{11} & a_{12} & 0 \\
0 & a_{22} & 0 \\
-1 & 0 & 0
\end{array}\right], \mathbf{B}:=[0,1,0]^{\top}, \\
\mathbf{K} & :=\left[K_{1}, K_{2}, K_{3}\right]^{\top}, \mathbf{E}:=\left[0, b K_{P}, 1\right]^{\top} .
\end{aligned}
$$

The system (15) is quadratically stabilizable if there exists a state-feedback gain $\mathbf{K}$ such that (15) is quadratically stable or equivalently if there exists a Lyapunov function $V(\boldsymbol{x})=\boldsymbol{x}^{\top} \mathbf{Q} \boldsymbol{x}$ with $\mathbf{Q}$ symmetric positive matrix $(\mathbf{Q}>\mathbf{0}$ in short) such that the time derivative of $V(\boldsymbol{x})$ is strictly negative for all $\boldsymbol{x}$ out of the origin. As pretty well-known, such a condition correspond to find a gain matrix $\mathbf{K}$ such that $(\mathbf{A}-\mathbf{B K})^{\top} \mathbf{Q}+\mathbf{Q}(\mathbf{A}-\mathbf{B K})$ is negative definite, i.e.,

$$
(\mathbf{A}-\mathbf{B K})^{\top} \mathbf{Q}+\mathbf{Q}(\mathbf{A}-\mathbf{B K})<\mathbf{0} .
$$

As shown in (Boyd, El Ghaoui, Feron, \& Balakrishnan 1994) (see p. 100), (17) can be expressed in an equivalent way by pre- and post-multiplying for $\mathbf{Q}^{-1}$ as follows

$$
\mathbf{Q}^{-1} \mathbf{A}^{\top}-\mathbf{Q}^{-1} \mathbf{K}^{\top} \mathbf{B}^{\top}+\mathbf{A} \mathbf{Q}^{-1}-\mathbf{B} \mathbf{K} \mathbf{Q}^{-1}<\mathbf{0},
$$

and, after using $\mathbf{P}:=\mathbf{Q}^{-1}$ and $\mathbf{Y}:=\mathbf{K P}$, via the following LMI

$$
\mathbf{P A}^{\top}-\mathbf{Y}^{\top} \mathbf{B}^{\top}+\mathbf{A P}-\mathbf{B Y}<\mathbf{0} .
$$

After solving (19), we obtain the gain $\mathbf{K}=\mathbf{P}^{-1} \mathbf{Y}$ and thus, from (14), the values of $K_{P}, K_{I}$, and $K_{D}$.

\subsection{Autopilot}

Likewise for the speed pilot, the yaw equation is decoupled and linearized around the desired advance speed $u_{d}$; the resulting system for yaw and rudder dynamics is:

$$
\begin{aligned}
\dot{\psi} & =r, \\
\dot{r} & =\frac{m x_{G} u_{d}+N_{r}}{I_{z}-N_{\dot{r}}} r+\frac{N_{\delta}}{I_{z}-N_{\dot{r}}} \delta, \\
\dot{\delta} & =\tau_{\delta}\left(\delta_{r}-\delta\right),
\end{aligned}
$$

where $\tau_{\delta}$ is a positive time constant accounting for the response of the rudder and $N_{\delta}$ is the rudders moment. After introducing the error $e:=\psi_{d}-\psi$ in (20) as augmented state variable with $\psi_{d}$ denoting the desired heading and proceeding as in Section 3.1 to account for a PID regulator, we obtain the system:

$\dot{\boldsymbol{x}}=(\mathbf{A}-\mathbf{B K}) \boldsymbol{x}+\mathbf{E} \psi_{d}$,

where $\boldsymbol{x}:=[\psi, r, \delta, e]^{T} \in \mathbb{R}^{4}$,

$$
\begin{aligned}
& \mathbf{A}=\left[\begin{array}{cccc}
0 & a_{12} & 0 & 0 \\
0 & a_{22} & a_{23} & 0 \\
0 & 0 & a_{33} & 0 \\
-1 & 0 & 0 & 0
\end{array}\right], \quad \mathbf{B}=[0,0,1,0]^{\top}, \\
& \mathbf{K}=\left[K_{1}, K_{2}, K_{3}, K_{4}\right]^{\top}, \quad \mathbf{E}=\left[0,0, b K_{P}, 1\right]^{\top},
\end{aligned}
$$

with

$$
a_{12}=1, \quad a_{22}=\frac{m x_{G} u_{d}+N_{r}}{I_{z}-N_{\dot{r}}}, \quad a_{33}=\tau_{\delta},
$$

$a_{23}=\frac{N_{\delta}}{I_{z}-N_{\dot{r}}}$, 
and

$K_{1}=b K_{P}, \quad K_{2}=b a_{12} K_{D}$,

$K_{3}=-K_{\delta}, \quad K_{4}=-b K_{I}$.

Note that $K_{\delta}$ accounts for a necessary feedback of the actuated rudder angle. Following the same reasoning from (17) to (19), we obtain the corresponding LMI and single out the PID coefficients.

\section{ENVIRONMENT}

Environmental forces and moments $\boldsymbol{\tau}_{E}$ are computed as the superposition of wind and sea actions.

\subsection{Wind Disturbances}

The resultant force and moment generated by the wind action are modeled as:

$\boldsymbol{\tau}_{W}=\left[\begin{array}{c}X_{A D_{W}} \cos \left(\gamma_{r_{w}}\right) \\ Y_{A D_{W}} \sin \left(\gamma_{r_{w}}\right) \\ N_{A D_{W}} \sin \left(2 \gamma_{r_{w}}\right)\end{array}\right] V_{R}^{2}\left(1+\frac{V_{G}}{V_{W}}\right)$,

where $X_{A D_{W}}, Y_{A D_{W}}, N_{A D_{W}}$ are the longitudinal, lateral force, and the moment wind coefficients, respectively ; $\gamma_{r_{w}}:=\psi_{W}-\psi$ is the relative angle between the vessel bow and the main incoming wind direction; $V_{W}$ is the wind mean speed; $V_{R}:=$ $\sqrt{\left(V_{W} \cos \gamma_{r_{w}}-u\right)^{2}+\left(V_{W} \sin \gamma_{r_{w}}-v\right)^{2}}$ is the relative mean wind speed; $V_{G}$ is the velocity of gusts whose time histories are reconstructed by Davenport spectrum approximation.

\subsection{Wave Disturbances}

Wave drift forces and moments are described by

$\boldsymbol{\tau}_{S}=\left[\begin{array}{c}X_{A D_{S}} \cos \left(\gamma_{r_{s}}\right) \\ Y_{A D_{S}} \sin \left(\gamma_{r_{s}}\right) \\ N_{A D_{S}} \sin \left(2 \gamma_{r_{s}}\right)\end{array}\right] H_{S}^{2}$,

where $X_{A D_{W}}, Y_{A D_{W}}, N_{A D_{W}}$ are respectively the longitudinal, lateral forces and the moment coefficients; $\gamma_{r_{s}}:=\psi_{S}-\psi$ is the relative angle between the vessel bow and the main wave incoming direction; $H_{S}$ is the significant wave height, whose time history is computed by an approximation of the JONSWAP spectrum.

\section{DELIVERED FORCES}

Delivered forces and moments are computed as follows:

$\boldsymbol{\tau}_{D}=\boldsymbol{\tau}_{P}+\boldsymbol{\tau}_{R}$,

where $\tau_{P}$ is the contribution due to the propulsion and $\tau_{R}$ denotes rudder effects.

\subsection{Propellers}

In navigation, portside and starboard propellers are coupled, then they are supposed to generate identical thrusts. Moreover, lateral propeller force is negligible.

As already mentioned, the only degree of freedom for surge speed control is the shaft speed. The total thrust array is then given by:

$\boldsymbol{\tau}_{P}:=\left[T_{p t}+T_{s b}, 0,0\right]^{\top}$,

where:

$T_{p t}=T_{s b}=K_{T}(J) \rho n_{p}^{2} D^{4}$

and

$J=\frac{u(1-w)}{n_{p} D}$

denotes the advance coefficient.

\subsection{Rudders}

Rudder forces and moment are modeled by means of drag and lift coefficients. The resulting array is described as:

$\boldsymbol{\tau}_{R}:=\left[2 D, 2 L, 2 L x_{r u d}\right]^{\top}$,

with

$L=\frac{1}{2} \rho A_{R} U^{2} C_{L}$,

$D=\frac{1}{2} \rho A_{R} U^{2} C_{D}$,

where $A_{R}$ is the rudder area; $U:=\sqrt{u^{2}+v^{2}} ; C_{L}$ and $C_{D}$ are functions of the rudder angle, computed in accordance to (SNAME 1989).

\section{SIMULATION RESULTS}

As mentioned in the Introduction, this case of study refers to a patrol vessel of the Italian Coast Guard. The speed pilot and autopilot will be installed on board within Summer 2015. Before on-board installation, the system is validated through several simulation tests. Simulation results of two specific maneuvers are reported with sea state 4 .

The first considered maneuver is presented in Section 6.1 and regards both the heading and speed control with bow quartering sea, wind, and current. This weather condition results to be one of the worst cases for the yaw control. The second maneuver in Section 6.2 aims to analyze head incoming disturbances that are more critical for the speed control. In both the cases, simulation results are satisfactory, errors being within the maximum allowed values. In the following figures, all motions are plotted as percentage of the maximum allowable errors, whereas thrusts and rudder angles are reported as percentage of their maximum deliverable quantities. 


\subsection{Bow-Quartering Sea}

In this section the first 10 minutes of simulation when all the incoming environmental disturbances are coming from $45^{\circ}$ are reported. The vessel is supposed to be on the right course with the desired advance speed when both the controllers are turned on. Then, initial errors for vessel heading and velocity are null, initial thrusts are corresponding to the necessary thrusts for the desired speed, rudders are hard over.

Figure 2 shows heading and speed errors. They are within the desired range. Figure 3 illustrates the transient behavior in the presence of disturbances (note the quick convergence of both yaw and speed errors).

The output of the heading controller is shown in Figure 4 in terms of required rudder angle (dotted line), vs the saturated one. Instead, saturation concerns upper and lower bounds of the deliverable angle and rate limiters. The first plot illustrates the whole time history where it is shown that a stable angle compensating disturbances is reached. The second plot focuses on the beginning of the maneuver and show that only rate limiters are active without saturation of the amplitude signals, as maximum allowable rudder angles are not involved and the controllers are able to stabilize the system only working in the linear range.

Figure 5 shows the output of the surge controller in terms of required shaft speed (dotted line) vs the saturated one. The second plot outlines the controller transient.

Figure 6 shows the disturbance time history; due to the main incoming direction of the aligned sea and wind, longitudinal, and lateral forces are of the same order of magnitude; in such a situation the moment reaches its maximum value.
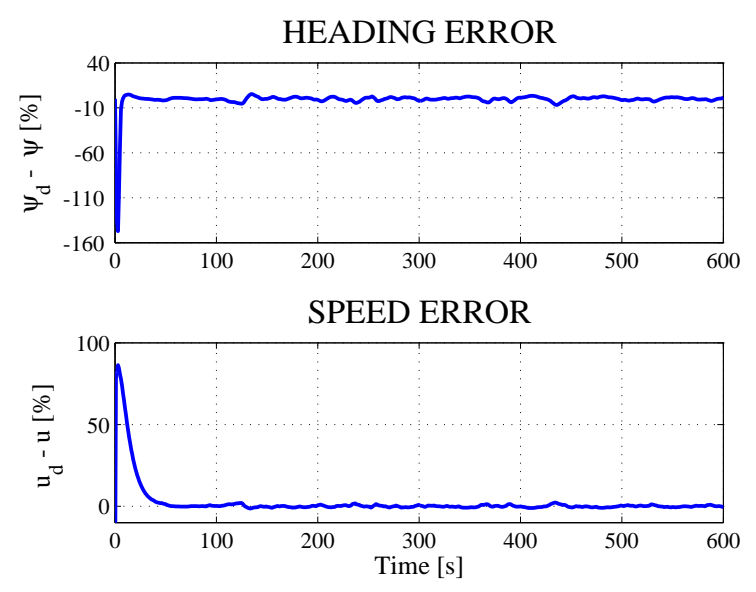

Figure 2: Heading and speed error for bow quartering sea.
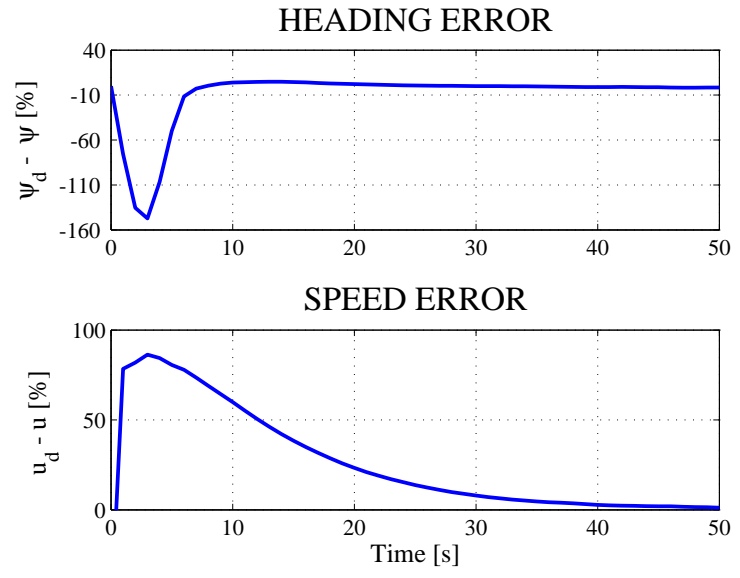

Figure 3: Sketch of the heading and speed error transient.
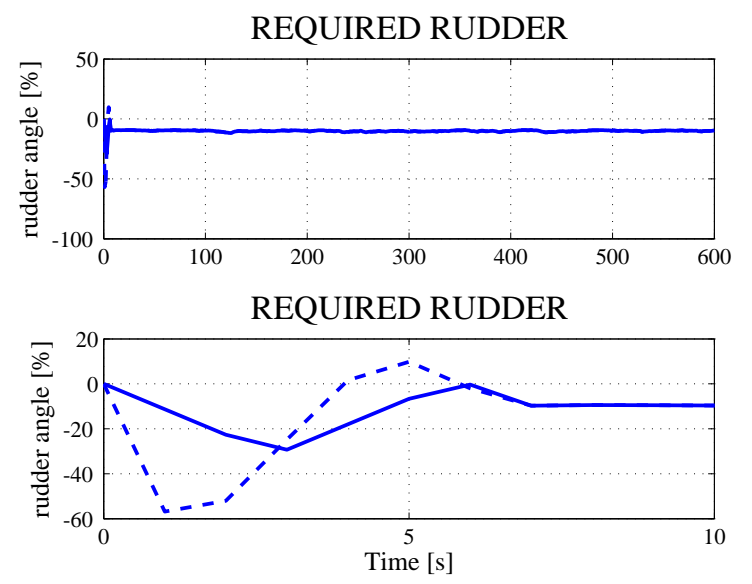

Figure 4: Rudder angle required for bow quartering sea.
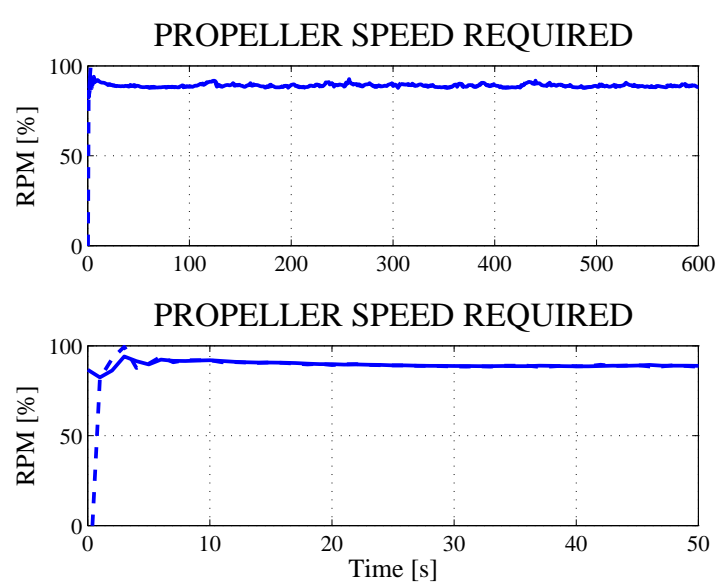

Figure 5: Shaft speed time history for bow quartering sea. 

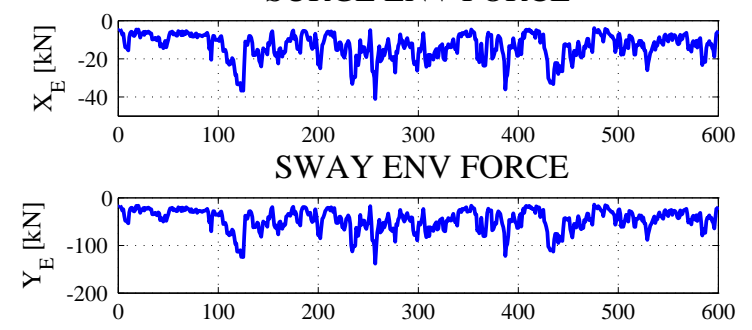

ENV MOMENT

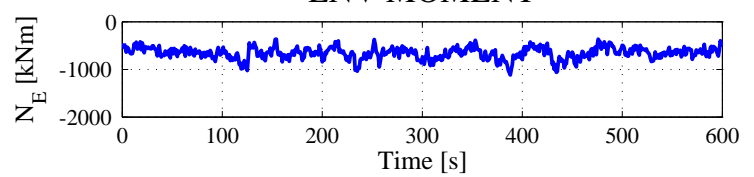

Figure 6: Environmental disturbances for bow quartering sea.

\subsection{Head Seas}

In this case environmental disturbances are coming from $5^{\circ}$ w.r.t. the bow, which is a challenging situation from the speed keeping point of view.

In figure 7, heading and speed errors are plotted. Due to the lower value of the disturbance moment, in the transient heading error is smaller than in the previous maneuver. As can be seen in figure 8 , in the transient also the speed error appears to be lower than in the previous case. Such a behavior can be due to a smaller rudder induced drag because of the lower rudder angles.

Figure 9 reports the required (dotted line) and delivered rudder angles. In this case, such records are overlying because, due to the small requirements, the rudder is instantaneously able to deliver the necessary angle.

Figure 10 shows the speed pilot output time history (dotted line) and the required shaft speed. The maneuver starts with the desired speed (the initial speed error is null) and then the speed pilot control is activated. In the transient from manual to automatic control a phenomenon called bump could arise.
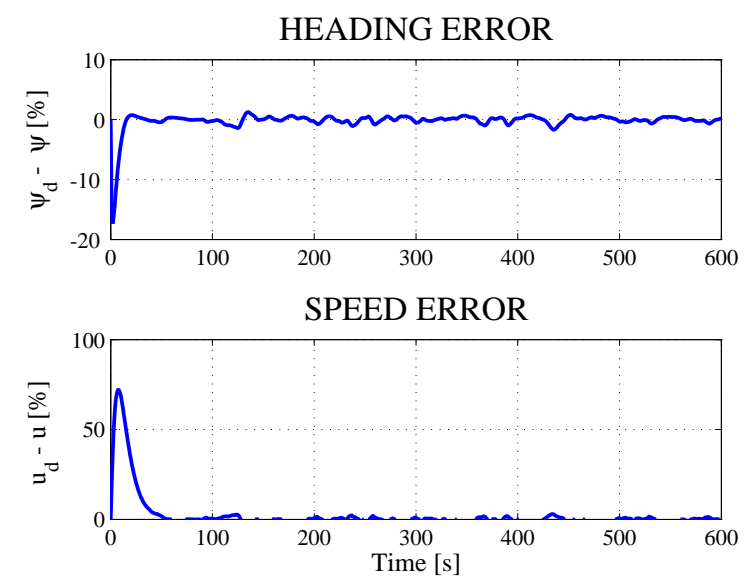

Figure 7: Heading and speed error for head sea.
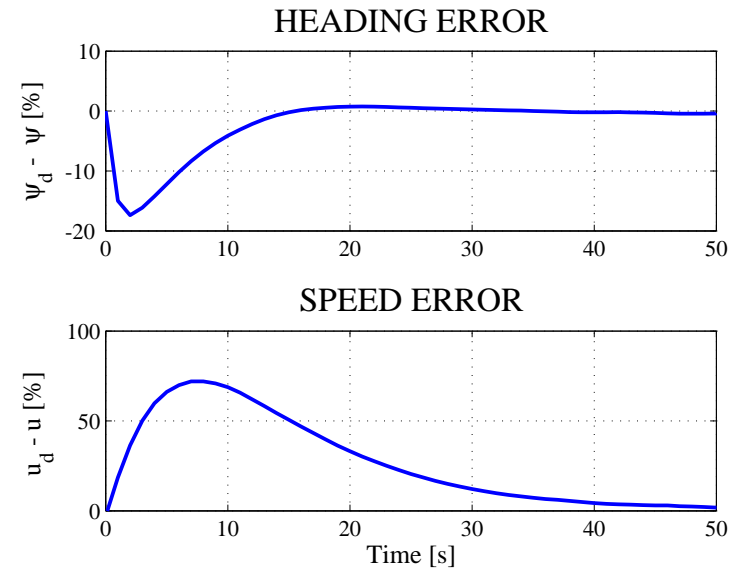

Figure 8: Sketch the heading and speed error transient.
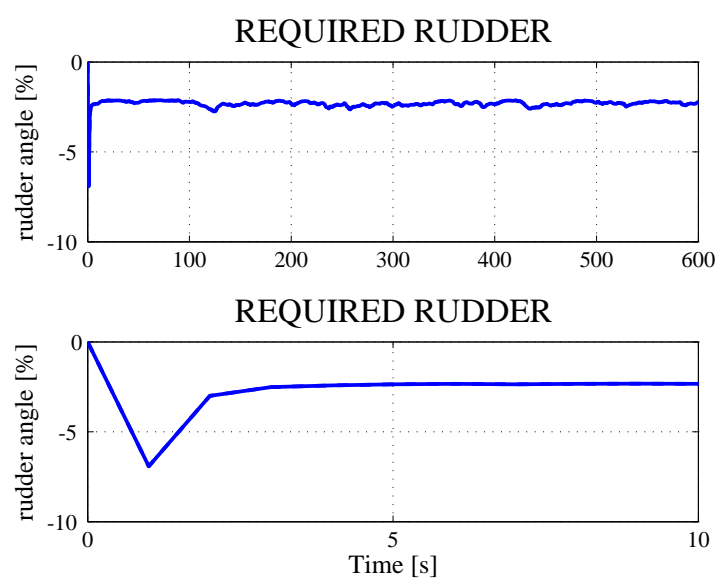

Figure 9: Rudder angle required for head sea.

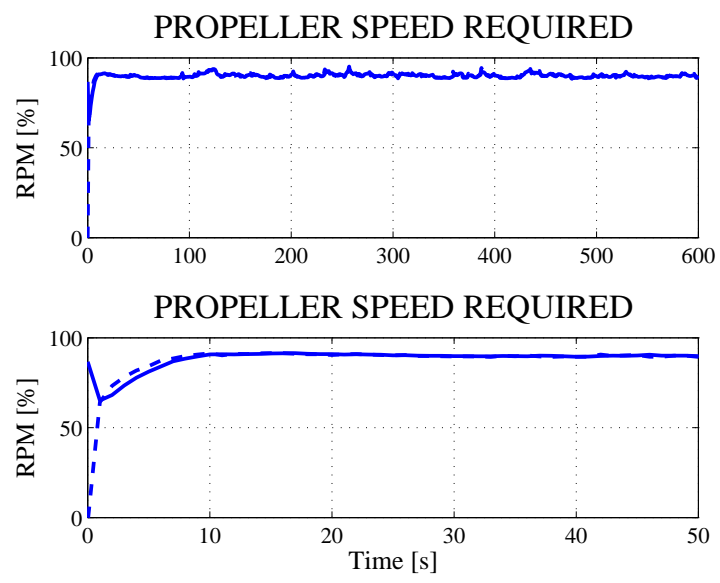

Figure 10: Shaft speed time history. 

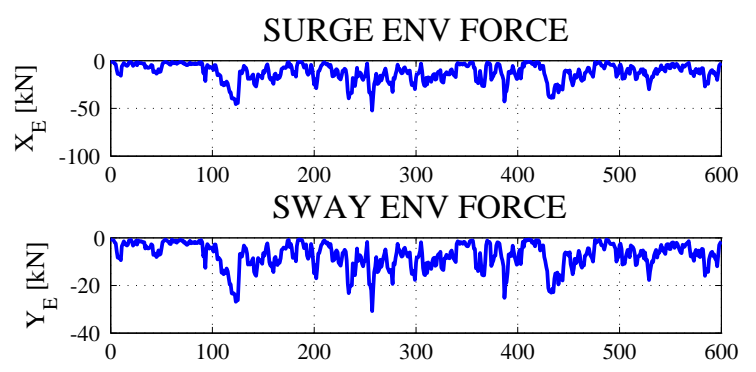

ENV MOMENT

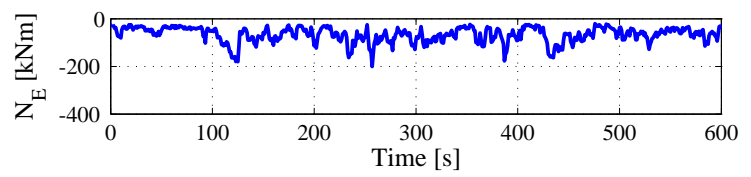

Figure 11: Environmental disturbances for head sea.

\section{CONCLUSION}

We have presented an approach to heading and speed control based on an "ad hoc" developed model. This study is motivated by both robustness evaluation and the request for most naval vessels to improve power management and cut fuel consumption without decreasing performance and, at the same time, reducing manning. Simulation results have been provided to illustrate the effectiveness of the proposed technical choices.

After the model calibration through maneuverability trials results, future work will concern the design of some additional controller functionality such as course keeping. Moreover, we will study techniques to reduce and possibly avoid bump effects.

\section{REFERENCES}

Altosole, M., D. Boote, S. Brizzolara, \& M. Viviani (2013). Integration of numerical modeling and simulation techniques for the analysis of towing operations of cargo ships. International Review of Mechanical Engineering (IREME) 7(7), 1236-1245.

Ankudinov, V., P. Kaplan, \& B. Jacobsen (1993). Assessment and principal structure of the modular mathematical model for ship maneuverability prediction and real-time maneuvering simulations. In Proc. International Conference on Marine Simulation and Ship Manoeuvrability, St. John's, Newfoundland, Canada.

Boyd, S., L. El Ghaoui, E. Feron, \& V. Balakrishnan (1994). Linear Matrix Inequalities in System and Control Theory, Volume 15 of Studies in Applied Mathematics. Philadelphia, PA: SIAM.

Fossen, T. (2002). Marine Control System. Norway, Trondheim: Marine Cybernetics.

Fuller, A. (1979, September). A history of control engineering 1800-1930. Electronic and Power 25(9).

Källström, C. (2000). Autopilot and track-keeping algorithms for high-speed craft. Control Engineering Practice 8(2), 185-190.

Lauvdal, T. \& T. Fossen (1998). Robust adaptive ship autopilot with wave filter and integral action. Int. Journal of Adaptive Control and Signal Processing 12(8), 605622.

Martelli, M., M. Viviani, M. Altosole, M. Figari, \& S. Vignolo (2014). Numerical modelling of propulsion, control and ship motions in 6 degrees of freedom. Proceedings of the Institution of Mechanical Engineers 228(4), 373-397.
Roberts, G., R. Sutton, A. Zirilli, \& A. Tiano (2003). Intelligent ship autopilots. a historical perspective. Mechatronics 13(10), 1091 - 1103.

SNAME (1989). Principle of Naval Architecture, Second Revision, Volume II. 601 Pavonia Avenue, Jersey City, NJ: Edward V. Lewis, Editor.

Tzeng, C.-Y. \& K.-F. Lin (2000). Adaptive ship steering autopilot design with saturating and slew rate limiting actuator. Int. Journal of Adaptive Control and Signal Processing 14(4), 411426. 